\title{
A Comparison of Epidemiological Characteristics of Central Nervous System Tumours in China and Globally from 1990 to 2019
}

\author{
Bo Zhu ${ }^{a}$ Xiaomei Wu ${ }^{b}$ Haozhe Piao $^{a}$ Shuang Xu ${ }^{c}$ Bing Yao ${ }^{a}$ \\ aDepartment of Neurosurgery, Cancer Hospital of China Medical University/Liaoning Cancer Hospital \& Institute, \\ Shenyang, China; ${ }^{b}$ Department of Clinical Epidemiology and Center of Evidence Based Medicine, The First Hospital \\ of China Medical University, Shenyang, China; ' Cibrary of China Medical University, Shenyang, China
}

\section{Keywords}

Central nervous system tumours · Epidemiology ·

Long-term trend - China - Disease burden

\begin{abstract}
Background: Despite their great disease burden, there have been few studies on the epidemiology of central nervous system tumours (CNSTs) in China. We used the latest data updated by GBD to analyse the trends of incidence, mortality, and disability-adjusted life years (DALYs) for CNSTs in China versus globally. Methods: Epidemiological data on CNSTs were extracted from GBD 2019. We used Joinpoint regression analysis to calculate the magnitude and direction of the trends and the age-period-cohort method to analyse the age, period, and cohort effects of the trend. Results: From 1990 to 2019 , the $106.52 \%$ increase in Chinese incident cases was higher than the global increase $(94.35 \%)$. The $67.32 \%$ increase in cancer deaths and $16.03 \%$ increase in DALYs were lower than the global increases (cancer death: 76.36\%; DALYs: 40.06\%). The age-standardized incidence rates (ASIRs) in China were higher than the global ASIRs, and the increase in China was higher than that globally. Although the age-standardized mortality rates and age-standardized
\end{abstract}

karger@karger.com www.karger.com/ned

Karger"

BOPEN ACCESS
(C) 2021 The Author(s)

Published by S. Karger AG, Basel

This is an Open Access article licensed under the Creative Commons Attribution-NonCommercial-4.0 International License (CC BY-NC) (http://www.karger.com/Services/OpenAccessLicense), applicable to the online version of the article only. Usage and distribution for commercial purposes requires written permission.
DALY rates in China were higher, their increases in China were less than those globally. Both in China and globally, the number and incidence, mortality, and DALYs by age group showed a bimodal distribution (younger than 5 years and older), and the peak in the older age group showed a backwards trend. The proportion of incident cases, cancer deaths, and DALYs also increased in the older age group. In the ageperiod-cohort model, the local drifts in the older age group were above zero. Conclusions: The burden of CNSTs is very serious in China, and we should pay attention to the key populations, early diagnosis technology, improvements in medical technology, and ways to reduce medical costs. We believe our results could help policymakers allocate resources efficiently to reduce the burden of CNSTs.

(C) 2021 The Author(s)

Published by S. Karger AG, Basel

\section{Introduction}

Due to their location, central nervous system tumours (CNSTs) can directly affect human health, especially physiological changes, including cognitive decline, growth retardation, endocrine disorders, and infertility [1-3]. CNSTs are likely to lead to cancer complications, 
placing a huge burden on society and families [4]. Globally, there were $>300,000$ incident cases and $>250,000$ cancer deaths in 2020 [5]. A global study on CNSTs found that the epidemiological trend of CNSTs had obvious regional characteristics. Environmental risk factors, genetic risk factors, medical technology level, and other socioeconomic factors were the important reasons for the differences [6]. East Asia was the region with the most incident cases of CNSTs for both sexes. At present, disabilityadjusted life years (DALYs) caused by CNSTs have decreased by approximately $10 \%$ at the global level, but they have increased by $22.5 \%$ in less developed regions. Due to the large population, the incidence and mortality of CNSTs in China rank first in the world. According to WHO statistics, there were 79,575 incident cases and 65,204 cancer deaths in China, accounting for more than one-quarter of the world's incident cases and cancer deaths, which placed a great disease burden on China [3]. Therefore, it is very important to grasp the epidemiological trend of CNSTs in China.

At present, the USA has done a good job of creating CNST databases. According to the registration information, the Central Brain Tumour Registry of the United States (CBTRUS) statistical report is the largest histologyspecific aggregation of CNST cases in the world that has structured and detailed registration definitions and is regularly published every 5 years. It performs a detailed analysis of the epidemiological trends, distribution of CNSTs types, and related factors (e.g., age, sex, and period) every 5 years [7]. From the recent CBTRUS Statistical Report, in the $0-14$ age group, the incidence of CNSTs exceeded that of leukaemia, becoming the most common tumour in this age group [7]. Recently, some European countries also detailed the epidemiological trends and characteristics of CNSTs and proposed targeted medical measures [8,9]. Our neighbouring South Korea has also conducted targeted epidemiological studies on CNSTs [10]. However, compared to studies on lung cancer, breast cancer, gastric cancer, and other cancers [11-13], there are fewer CNST studies, especially in China. Due to the unknown epidemiological characteristics of CNSTs, this is a disadvantage for the formulation of targeted medical policies and measures, and the gap in the research depth on CNSTs in China is apparent. For the reasons given earlier, we carried out this study.

The trends of incidence, mortality, and DALY rates are important indicators to measure the disease burden over time. Understanding the epidemiological characteristics of CNSTs and analysing the causes of their long-term changes are very important for understanding the aetiol- ogy of CNSTs and formulating the corresponding medical measures. By using the latest data updated by GBD, compared to the global level, our study investigated the changes in CNST epidemiology and the related factors in China to provide basic information for aetiological research and the formulation of medical measures for CNSTs.

\section{Methods}

The data sources for GBD were mainly systematic reviews, survey data, disease registries, hospital administrative data, claims, inpatient and outpatient data, and case notifications, which were organized by the Guidelines for Accurate and Transparent Health Estimates Reporting statement [14]. The Guidelines for Accurate and Transparent Health Estimates Reporting defined the best practices for documenting studies that synthesize evidence from multiple sources to quantitatively describe past and current population health and its determinants [15]. These practices included documenting and sharing data inputs, analyses and methods, and results.

We performed a secondary database descriptive study using the GBD 2019 results. We extracted related data on CNST incidence, mortality, and DALYs in China and globally. The extracted 30year data on incidence, mortality, and DALYs were used to analyse the epidemiological trend of CNSTs, which mainly referred to the time trend. The related data are available to the public and can be extracted via the GBD Results Tool (http://ghdx.healthdata.org/ gbd-results-tool) [16]. We used data according to the GBD Protocol.

We used Microsoft Excel 2016 to organize the extracted data. The DisMod-MR 2.1 model, a Bayesian meta-regression method, was used for each indicator. We used Joinpoint software to analyse the long-term trend of the age-standardized rate (ASR) of the incidence, mortality, and DALYs of CNSTs from 1990 to 2019. The annual percentage change (APC) and the corresponding 95\% CIs were used to assess the magnitude and direction of trends over time. The APC was estimated by regression coefficients. The ASR showed an upwards trend when the APC and the lower CI limit were positive, and the ASR showed a descending trend when the $\mathrm{APC}$ and upper CI limit were negative. We further performed subgroup analysis on the age-standardized indicators of incidence, mortality and DALYs by sex, age, and year. We observed changes in the proportion of incidence, mortality, and DALYs in different age groups from 1990 to 2019.

We further explored the causes of the epidemiological trend in CNSTs and used the age-period-cohort model (APCM) to analyse the age, period, and cohort effects on the incidence, mortality, and DALYs. The purpose of APCM is to evaluate the impact of age, period, and cohort effects on related outcomes, such as the ASR, on the incidence, mortality, and DALYs in our study. The age effect represents different risks of the related outcomes in different age groups. The period effect represents the effect of the time change on the related outcomes of all age groups. The cohort effect represents the change in the related outcomes between individual groups (cohorts) with the same year of birth. The APCM could provide a useful parametric framework that complements stan- 


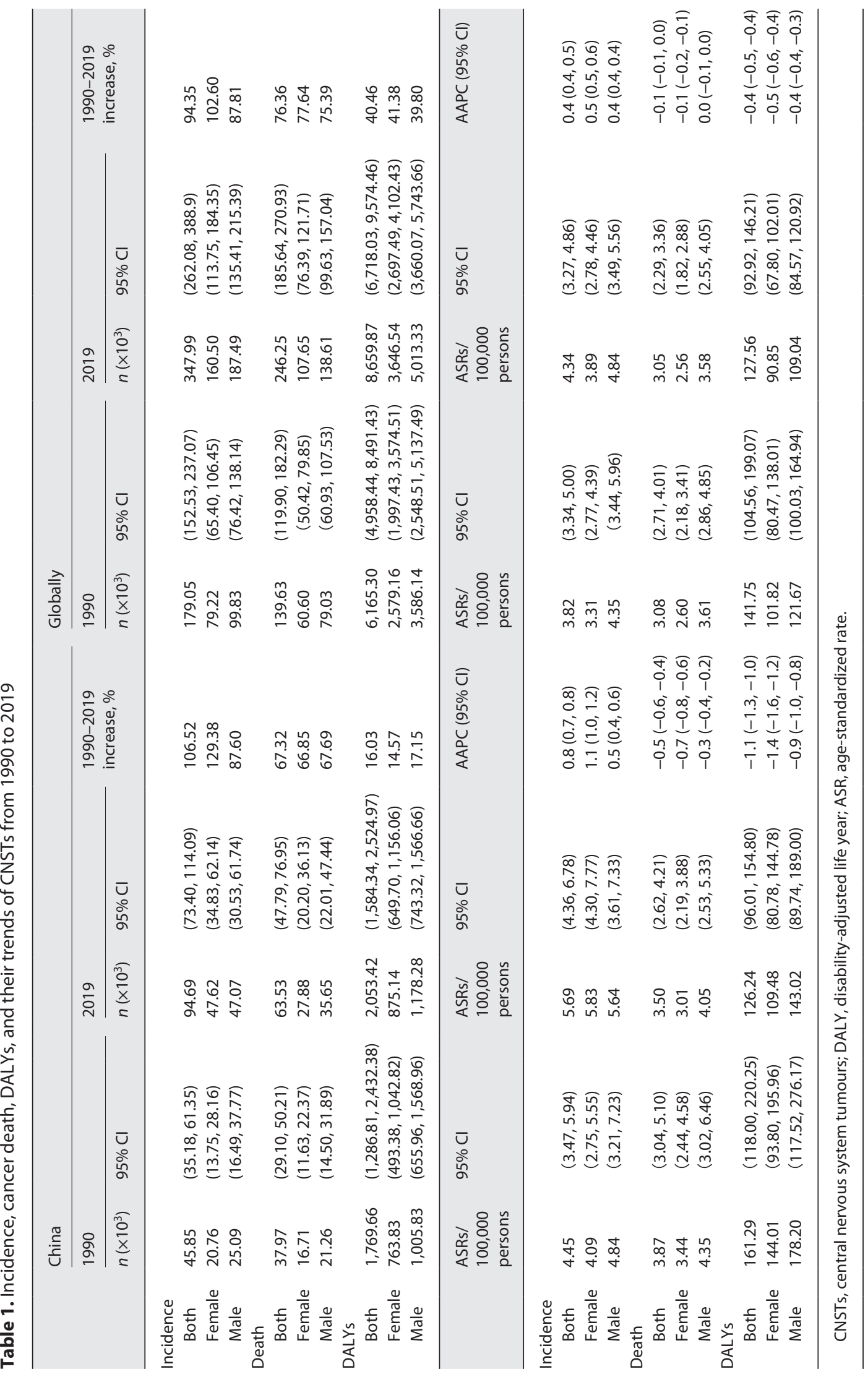




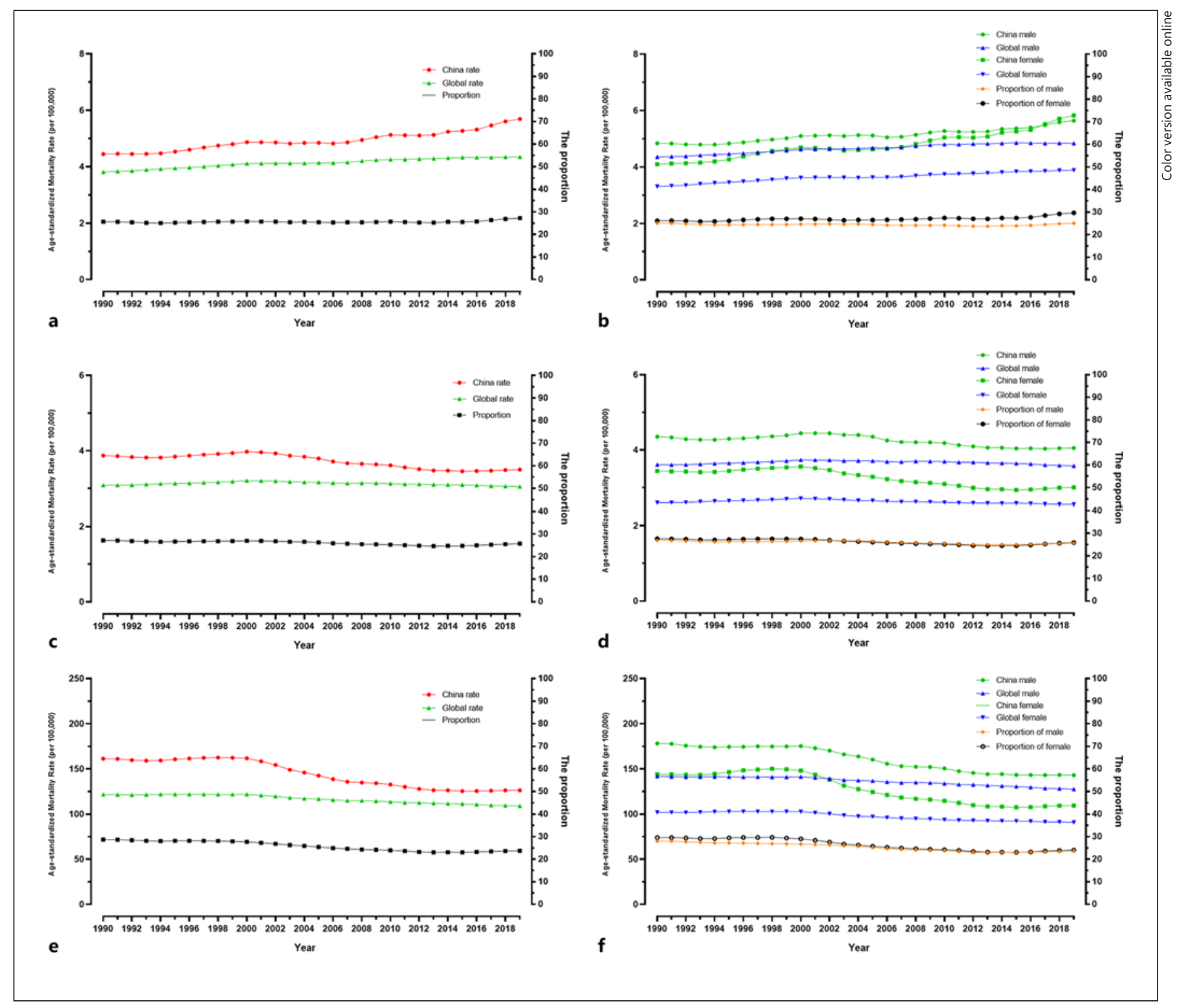

Fig. 1. ASR in incidence, mortality, and DALYs of CNSTs and the proportion of incident cases, cancer deaths, and DALYs in China and globally from 1990 to 2019. a ASIR of CNSTs and the proportion of incident cases for both sexes in China and globally from 1990 to 2019. b ASIR of CNSTs and the proportion of incident cases for males and females in China and globally from 1990 to 2019. c ASMR of CNSTs and the proportion of cancer deaths for both sexes in China and globally from 1990 to 2019. d ASMR of CNSTs and the proportion of cancer deaths for males and females

in China and globally from 1990 to 2019. e ASDR of CNSTs and the proportion of DALYs for both sexes in China and globally from 1990 to 2019. f ASDR of CNSTs and the proportion of DALYs for males and females in China and globally from 1990 to 2019. ASR, age-standardized rate; ASIR, age-standardized incidence rate; ASMR, age-standardized mortality rate; ASDR, age-standardized disability-adjusted life years rate; DALY, disability-adjusted life year; CNSTs, central nervous system tumours.

Fig. 2. Number of incident cases and incidence rate of CNSTs in each age group in China and globally (1990 vs. 2019). a The number of incident cases and incidence rate of CNSTs in each age group for both sexes in China and globally (1990 vs. 2019). b The number of incident cases and incidence rate of CNSTs in each age

group for females in China and globally (1990 vs. 2019). c The number of incident cases and incidence rate of CNSTs in each age group for males in China and globally (1990 vs. 2019). CNSTs, central nervous system tumours.

(For figure see next page.) 


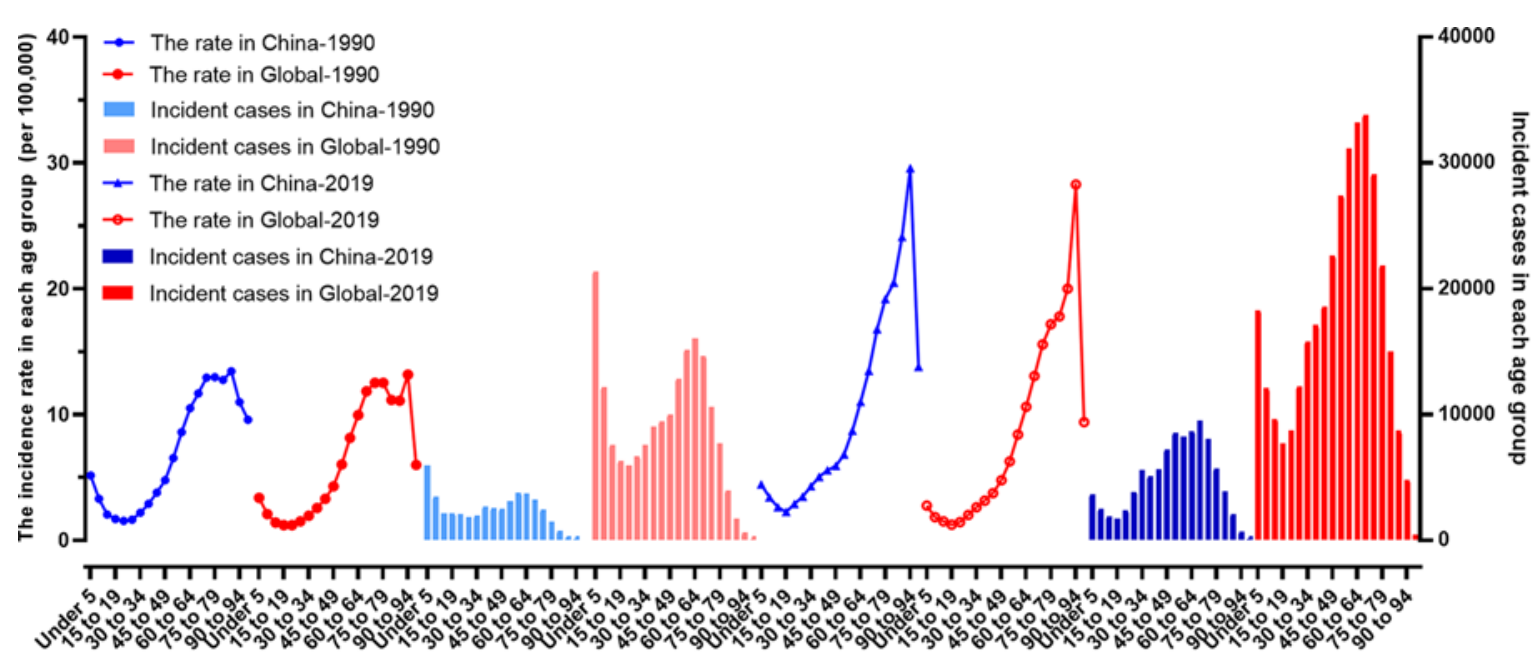

a

Age group

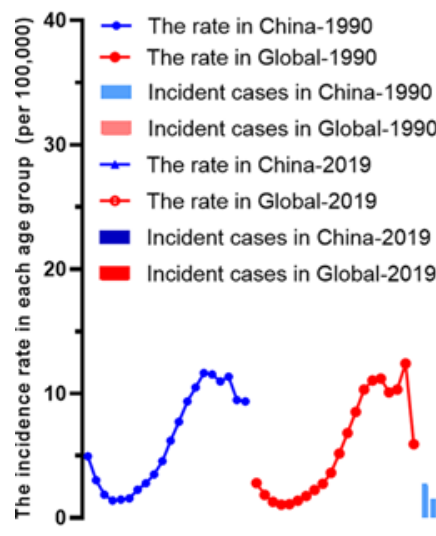

|huninul||I.... |||||||||||| .

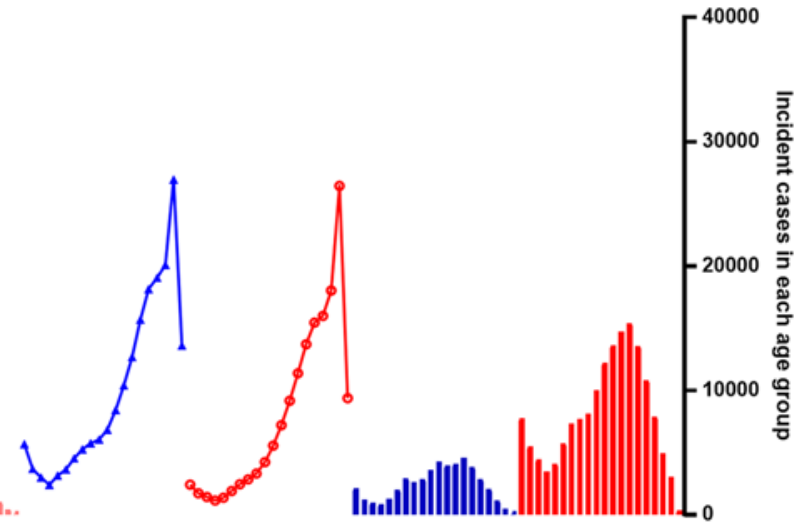

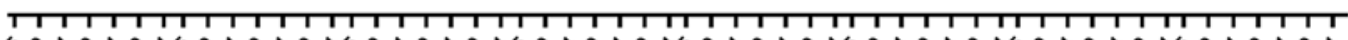

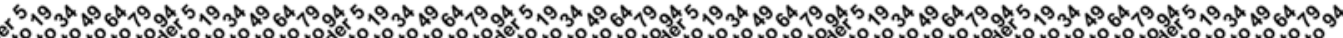
vis:

b

Age group

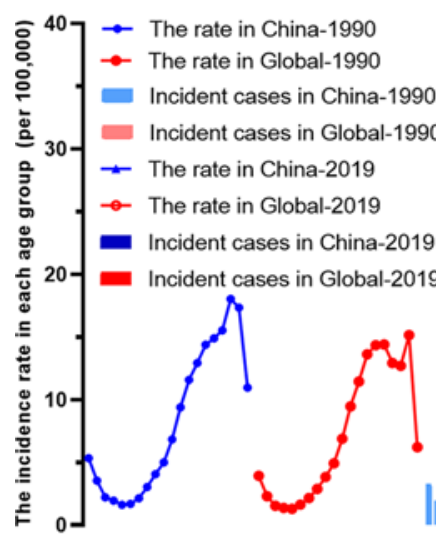

|lnul|ul||l.
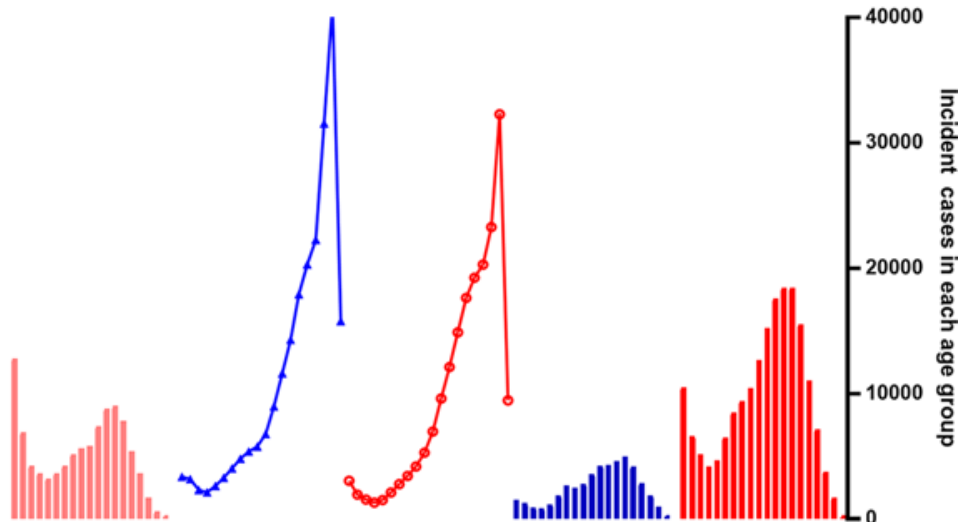

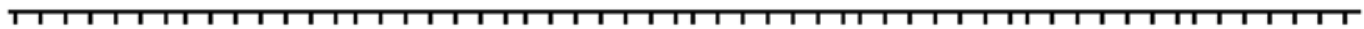

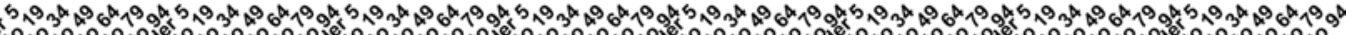
r.5:5\%

c

Age group

2 


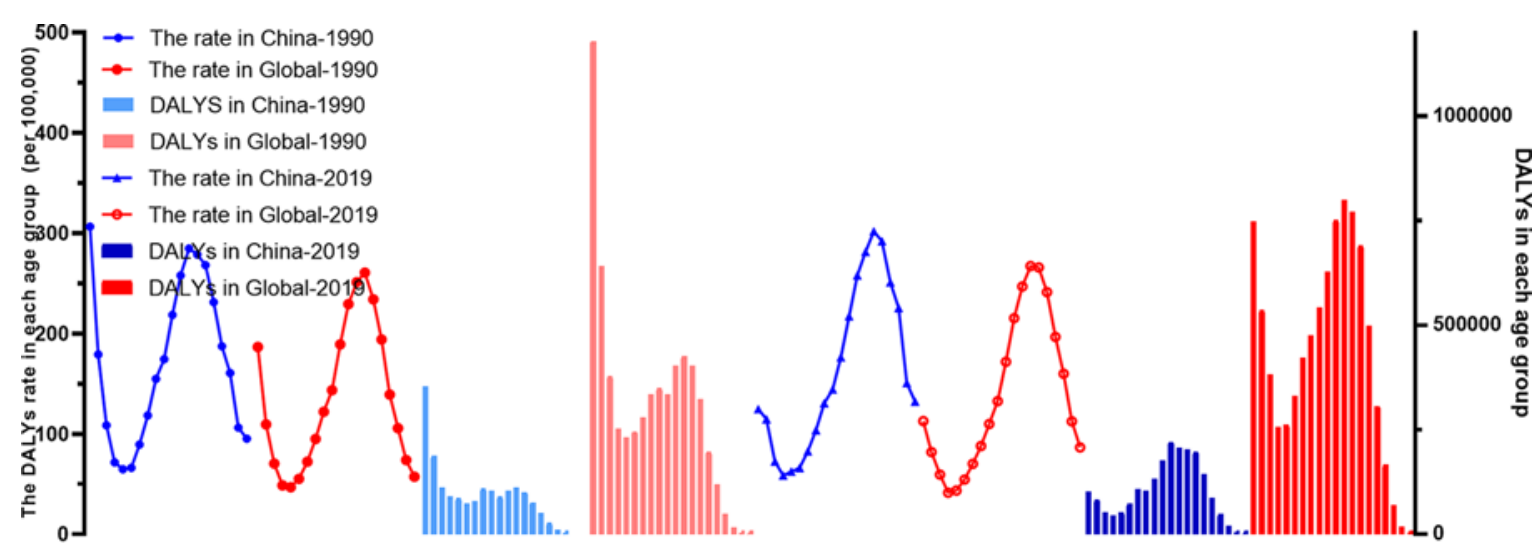

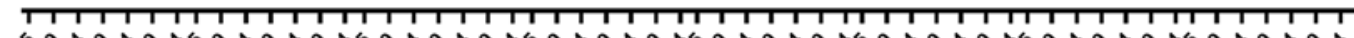

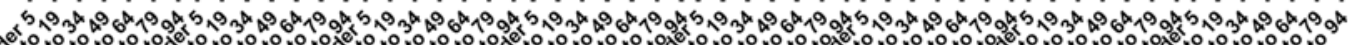
vis:

a

Age group

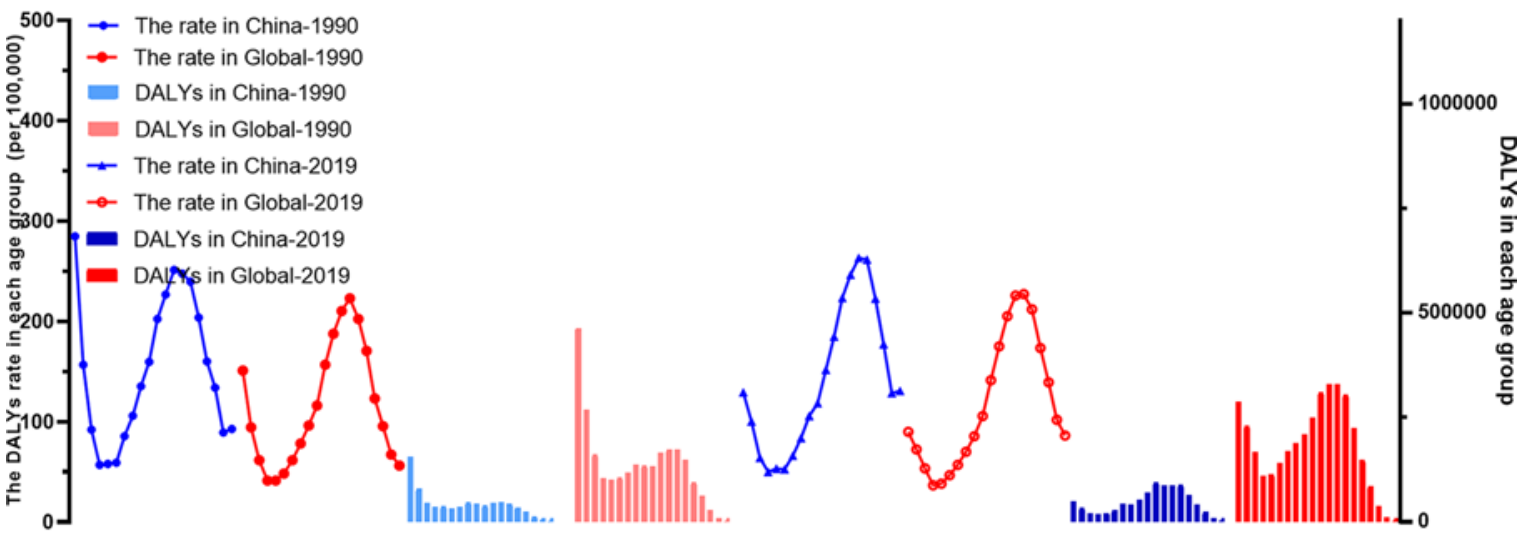

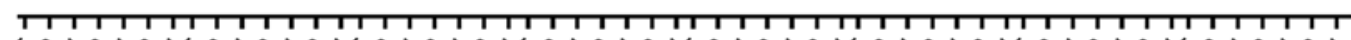

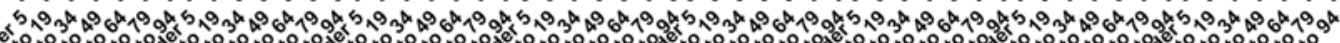
r.5.

b

Age group

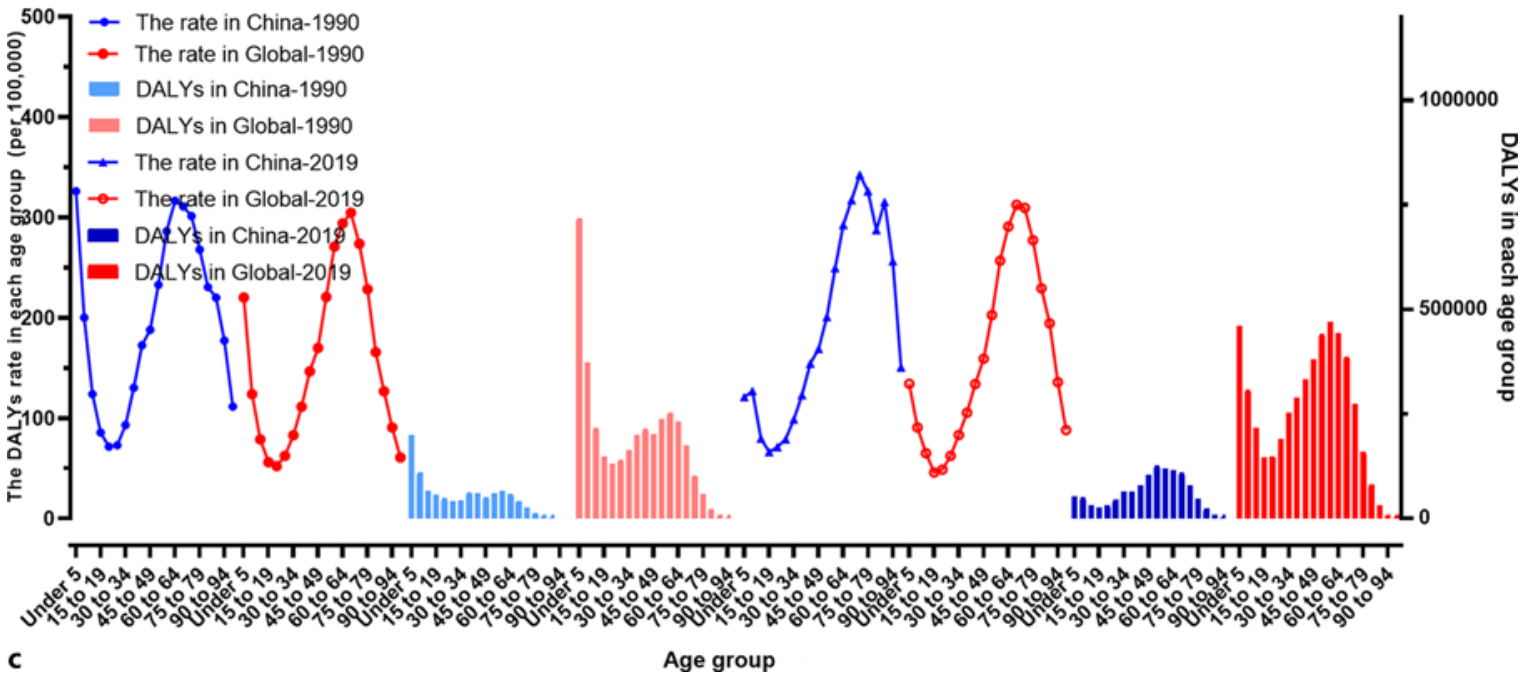

(For legend see next page.)

The Epidemiological Trend of CNSTs in China and Globally
Neuroepidemiology 2021;55:460-472 DOI: $10.1159 / 000519463$ 
dard nonparametric descriptive methods, which were described in detail in previous studies $[17,18]$. The model contains 3 aspects: the longitudinal age curve, period rate ratios, and cohort rate ratios [18]. Finally, we used local drifts and net drift to assess the APC of the expected age-adjusted rates over time. The "apc" package was used to perform APCM in R statistical software ( $\mathrm{R}$ version 3.5.1).

\section{Results}

Overall, the number of incident cases in China increased by $106.52 \%$ from 1990 to 2019, which was higher than the global increase (94.35\%). However, the number of cancer deaths and DALYs increased by $67.32 \%$ and $16.03 \%$ from 1990 to 2019 , respectively, which were lower than the global increase (cancer deaths: $76.36 \%$; DALYs: $40.06 \%$ ). In terms of sex, the number of female incident cases in China increased by $106.52 \%$ from 1990 to 2019 , which was higher than the global increase (102.60\%), while a similar increase in China $(87.60 \%)$ and globally $(87.81 \%)$ was observed in male incident cases. For cancer deaths and DALYs, the female and male increase in China was lower than the global increase. From 1990 to 2019, the age-standardized incidence rates (ASIRs) in China were always higher than the global ASIRs, and the APC in China was higher than the global APC. Although the agestandardized mortality rates (ASMRs) and age-standardized DALY rates (ASDRs) in China were always higher, they showed a downwards trend, and their decreases were higher than the global ASMRs and ASDRs. In terms of sex, similar changes in ASIRs, ASMRs, and ASDRs were observed for females and males, both in China and globally. The number of incident cases, cancer deaths, and DALYs accounted for $>25 \%$ worldwide (Table 1; Fig. 1).

In terms of age, the number of incident cases in China and globally showed a similar trend in 1990 and 2019 and had a bimodal distribution. In 1990, the 2 peaks were in

Fig. 3. The number and rate of DALYs of CNSTs in each age group in China and globally (1990 vs. 2019). a The number and rate of DALYs of CNSTs in each age group for both sexes in China and globally (1990 vs. 2019). b The number and rate of DALYs of CNSTs in each age group for females in China and globally (1990

Fig. 4. Age-period-cohort effects on the CNST incidence rate in China and globally. a Age effects on the CNST incidence rate for both sexes in China and globally. $\mathbf{b}$ Age effects on the CNSTs incidence rate for females and males in China and globally. c Period effects on the CNST incidence rate for both sexes in China and globally. d Period effects on the CNST incidence rate for females and males in China and globally. e Cohort effects on the CNST the under 5-year age group and the 60- to 64-year age group; in 2019, the 2 peaks were in the under 5 -year age group and the 65- to 69-year age group. For the incidence, in 1990, the 2 peaks in China were in the under 5 -year age group and the 85- to 89-year age group, and in 2019, the 2 peaks were the under 5 -year age group and the 90- to 94 -year age group. Globally, the 2 peaks in 1990 were in the under 5-year age group and in the 60- to 64-years age group; the 2 peaks in 2019 were in the under 5 -year age group and the 90- to 94-year age group (Fig. 2). A similar trend for age groups was observed for cancer deaths (online suppl. Fig. 1; for all online suppl. material, see www. karger.com/doi/10.1159/000519463). Although the number and rate of DALYs also showed a bimodal distribution, the 2 peaks were in different age groups. For the number of DALYs, in 1990, the 2 peaks in China and globally were in the under 5 years age group and the 55-59 years age group, and in 2019, the 2 peaks were in the under 5 years age group and the $50-54$ years age group. For the rate of DALYs, in 1990, the 2 peaks in China were in the under 5 years age group and the 60-64 years age group, and in 2019, the 2 peaks were in the under 5 years age group and the 70-74 years age group; in 1990, the 2 peaks globally were in under the 5 years age group and the 55-59 years age group, and in 2019, the 2 peaks were in the under 5 years age group and the 65-69 years age group (Fig. 3).

In China, in terms of the changes in the proportion of incident cases, cancer deaths and DALYs in each age group from 1990 to 2019, the changes in the incident cases and cancer deaths were similar. The proportions in the younger age groups (under 5 to 20 - to 24 -year age groups) decreased significantly, while the proportions in the older age groups (60-64 to 95 plus years age groups) increased significantly (online suppl. Fig. 2, 3); for DALYs, the proportions in the younger age groups (under 5 to $20-24$ years age groups) decreased significantly, while the

vs. 2019). c The number and rate of DALYs of CNSTs in each age group for males in China and globally (1990 vs. 2019). CNSTs, central nervous system tumours; DALY, disability-adjusted life year.

incidence rate for both sexes in China and globally. $f$ Cohort effects on the CNST incidence rate for females and males in China and globally. g Local drifts on the CNST incidence rate in each age group for both sexes in China and globally. $\mathbf{h}$ Local drifts on the CNST incidence rate in each age group for females and males in China and globally. CNSTs, central nervous system tumours; ASIR, age-standardized incidence rate; $R R$, rate ratio.

(For figure see next page.) 

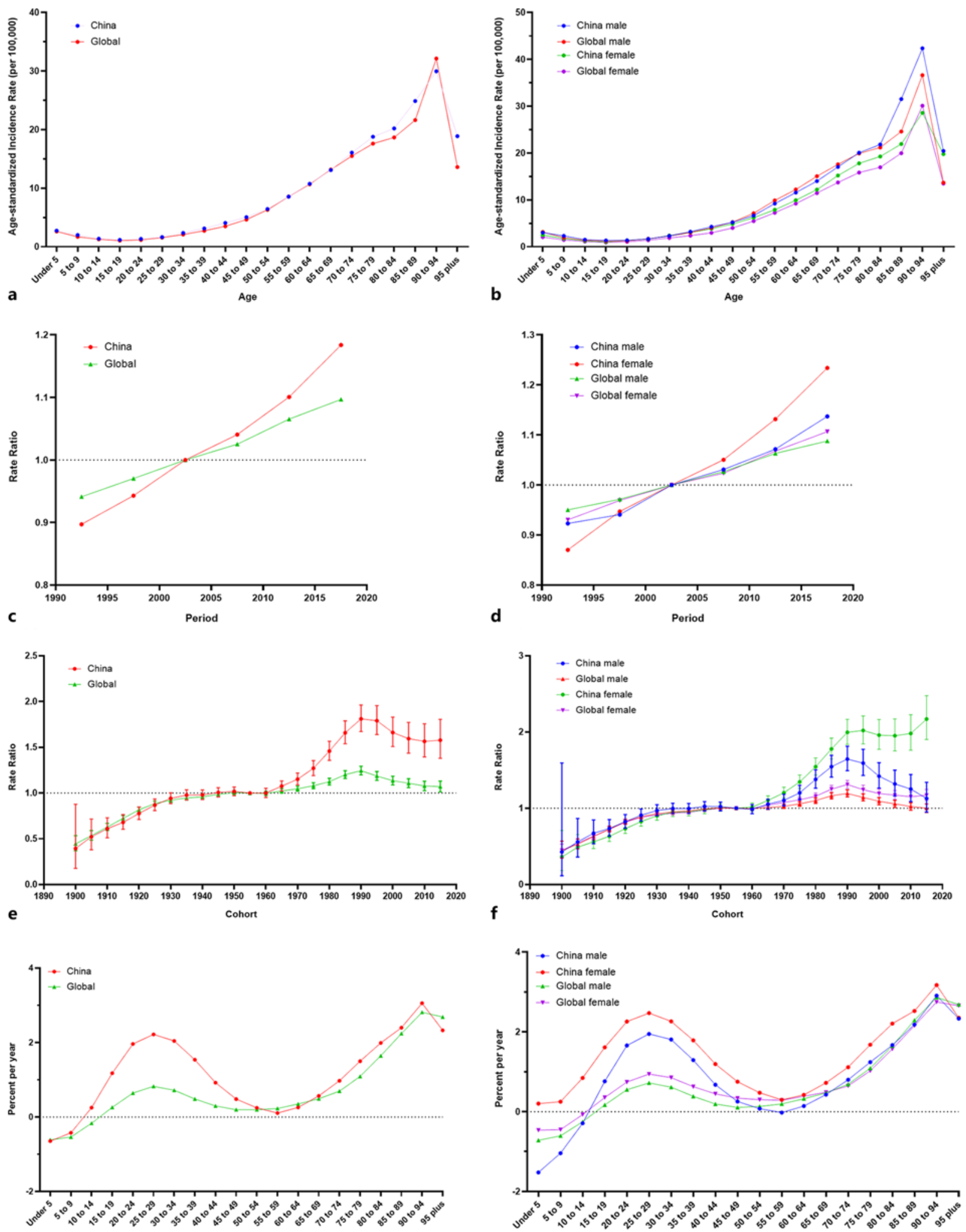

g

h

Age 

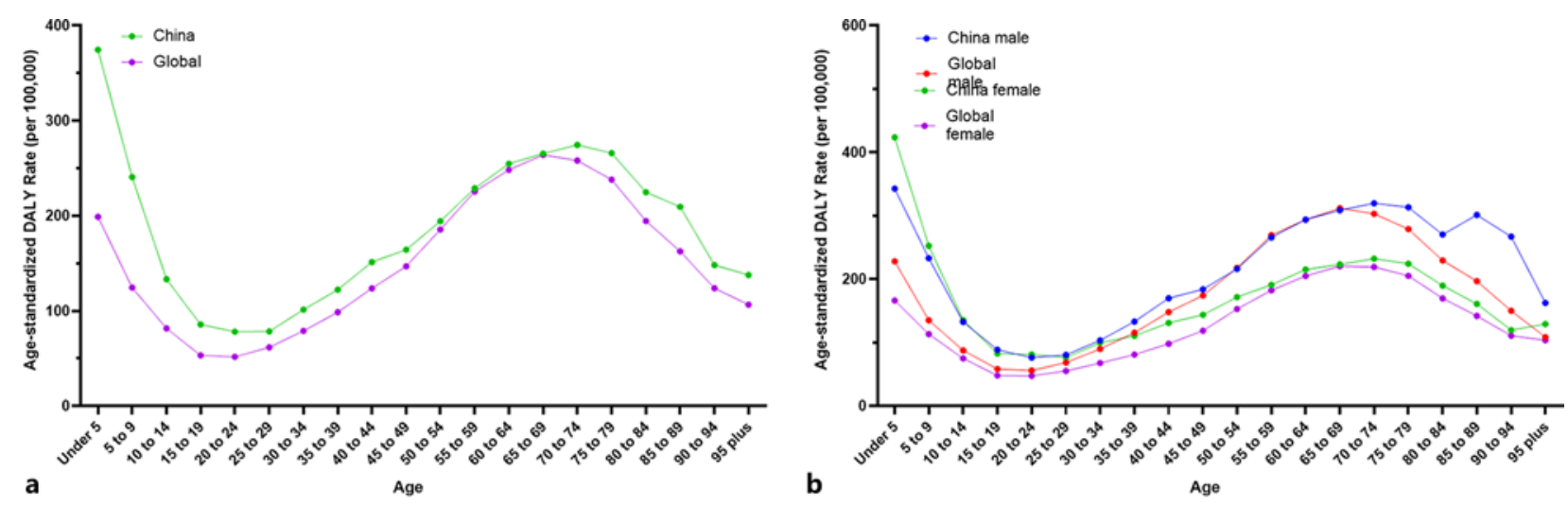

a
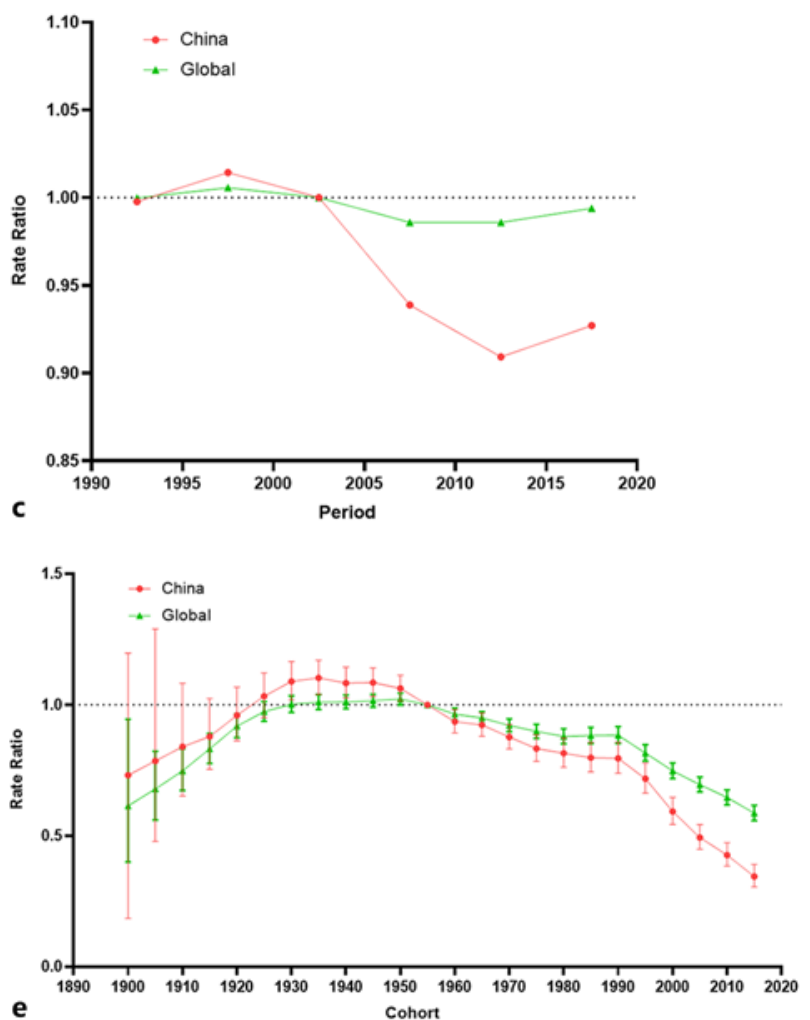

e

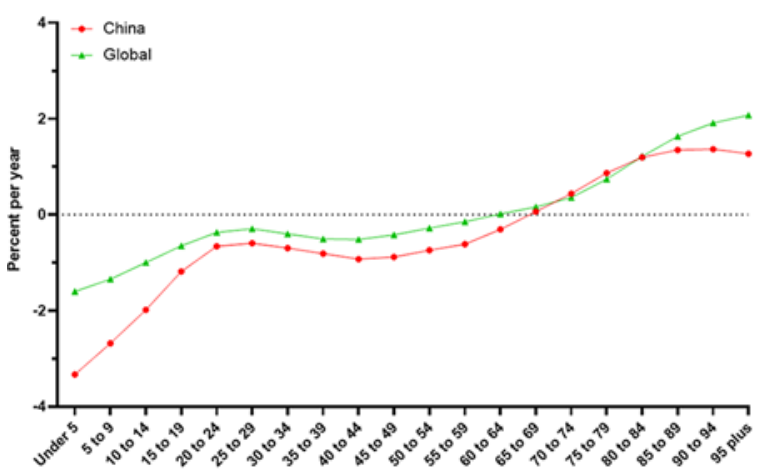

g
Age b
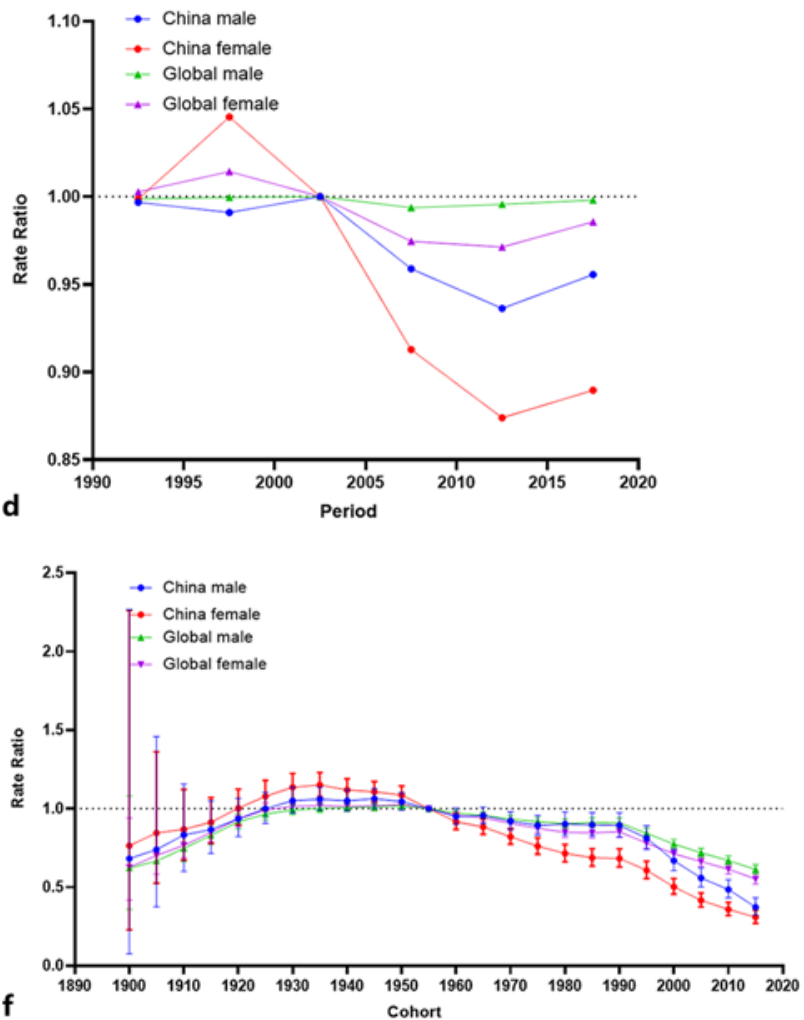

${ }^{4} 7 \rightarrow$ China male

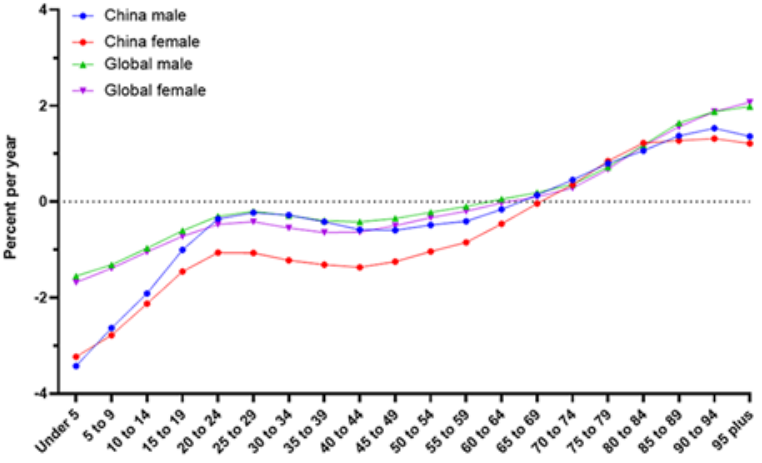

h 
proportions in the older age groups (45-49 to 95 plus years age groups) increased significantly (online suppl. Fig. 4) The changes were similar for both sexes. We also found similar trends for global cases (online suppl. Fig. 5-7).

The effects of age, period, and cohort on CNST incidence in China and globally are shown in Figure 4 . The age effects increased with increasing age, and similar trends were observed in males and females. The changes in the period effect in China and globally were similar; the period effect increased by years, and the increase in the period effect in China was higher than that globally. In terms of sex, an increase in Chinese females was also obvious. Overall, the cohort effect in China and globally showed an upwards trend. After 1990, the cohort effect was stable. In terms of sex, the cohort effect of females showed a wavy upwards trend after 1990, while that of males showed a downwards trend. The local drifts in China and globally showed risedecline-rise change curves. The 2 peaks of local drifts were in the 25- to 29- and 90- to 94-year age groups in China and globally. The values of local drifts in China were higher than those globally, and the increase in local drifts in China was also higher than that globally. In terms of sex, in China, the values of local drifts in females were higher than those in males in all age groups, and globally, the values of local drifts in females were higher than those in males before the 65- to 69-year age group.

In both China and globally, the changes in age, period, and cohort effect on CNST mortality and DALY rate were similar (see Fig. 5 and online suppl. Fig. 8). The age effects in China and globally showed decline-rise-decline change curves. The 2 peaks of age effects were in the under 5 - and 70 - to 74-year age groups in China and globally. The values of the age effects in China were higher than those globally, and the increase in age effects in China was also higher than that globally. In terms of sex, in China, the values of age effects in females were higher than those in males in all age groups, and globally, the values of age effects in females were higher than those in males older than the 65-69 years. The changes in the period effect in China and globally were similar. The period effect first increased and then decreased slightly, and the decrease in

Fig. 5. Age-period-cohort effects on the CNST DALYs rate in China and globally from 1990 to 2019. a Age effects on the CNST DALYs rate for both sexes in China and globally. $\mathbf{b}$ Age effects on the CNST DALYs rate for females and males in China and globally. c Period effects on the CNST DALYs rate for both sexes in China and globally. d Period effects on the CNST DALYs rate for females and males in China and globally. e Cohort effects on the
China was higher than that globally. In terms of sex, similar changes in the period effect for males and females were observed, and the decrease in females was the largest. The cohort effect first increased and then decreased gradually after 1940. The decrease in China was higher than that globally. The changes in cohort effects for males and females showed an overall trend. The local drifts of the DALY rate in China and globally first increased, then remained stable among the 20- to 24- to 50- to 54-year age groups and then increased among those older than the 55- to 59-year age group. Similar changes in local drifts were observed in males and females. In China, the values of local drifts in males were higher than those in females within the 10- to 14- to 60- to 64-year age groups, while globally, the values of local drifts in males and females were similar in all age groups.

\section{Discussion}

The results of our study showed that the disease burden of CNSTs in China and globally increased from 1990 to 2019, mainly due to the increase in incident cases, deaths, and DALYs. The ASIR in China and globally increased, the ASMR and ASDR decreased, and the increase in ASIR was higher than the decrease in ASMR and ASDR. This may be mainly due to timely diagnosis and treatment, as well as improvements in medical technology [19]. China experienced rapid development of its social economy, ranking at the forefront of the world in both GDP and its increase. Medical and health investments have increased yearly, and medical conditions have greatly improved, which are higher than the global average level. Moreover, with the progression of Chinese social civilization, awareness of sex equality has increased, and the protection of women's rights has been emphasized [20]. Therefore, compared with the former period, Chinese women have more medical resources, such as access to physical examinations, disease diagnosis, and treatment. Our results also showed that the increase in incidence and the decrease in mortality and DALYs in China were higher than those globally, and the increase

CNST DALYs rate for both sexes in China and globally. $\mathbf{f}$ Cohort effects on the CNST DALYs rate for females and males in China and globally. $\mathbf{g}$ Local drifts on the CNST DALYs rate in each age group for both sexes in China and globally. $\mathbf{h}$ Local drifts on the CNST DALYs rate in each age group for females and males in China and globally. CNSTs, central nervous system tumours; DALY, disability-adjusted life year; RR, rate ratio. 
in incidence and the decrease in mortality and DALYs in Chinese females were higher than those in males.

In contrast to other cancers, the incidence, mortality, and DALYs of CNSTs presented a bimodal distribution, which deserves attention in the control and treatment of CNSTs. There are 2 main reasons for the high incidence of CNSTs in childhood. Parents may suffer from diseases during the childbearing period or be exposed to physical and chemical factors for a long time prior to conception, which would have a significant impact on their offspring $[21,22]$. Similar to the occurrence of adult cancers, environmental pollution around newborns - especially easily neglected ionizing radiation, iatrogenic radiotherapy, chemical drugs, virus infection, immune deficiency, and even eating habits - has been related to the occurrence of cancers in children, especially cancers during school age and adolescence [23, 24]. In our study, from 1990 to 2019, the proportion of incident cases, cancer deaths, and DALYs during childhood gradually decreased, indicating that a series of measures focussing on children's health, such as prenatal examinations and neonatal screening, were effective. In adults, the peak age of incidence, death, and DALYs showed a backwards trend. The proportion of incident cases, cancer deaths, and DALYs in the older age group gradually increased from 1990 to 2019.

The treatment of CNSTs includes a multidisciplinary approach that combines biopsy or aggressive surgical resection with postoperative radiotherapy and chemotherapy. Patients need access to neurosurgical services, intensive care units, and highly specialized radiation and neuro-oncology services [25]. In recent years, with the development of medical technology, the extension of the average life expectancy, the improvement of living standards, and the increase in social security coverage, people are increasingly able to afford high-quality medical examinations and treatment costs of CNSTs, which may lead to backwards-moving peak age.

We further analysed the effects of age, period, and cohort on the long-term trends of CNST epidemiology. We also analysed the trends of incidence, mortality, and DALY rate in each age group older than 30 years. We found that the incidence and mortality rate of CNSTs were higher in the 60- to 80-year age group, which reminded us that age is one of the risk factors for CNSTs. This is consistent with the survey data from South Korea and slightly different from the data of the USA. In terms of the DALY rate, we can see that the DALY rate in the under 5 and 70-74 year age groups was higher due to the prolonged survival time and the increased medical cost. This suggests that we should not only attach importance to the diagnosis and treatment of elderly individuals but also pay attention to the pathogenesis and control of this disease in children. Although ionizing radiation is the only well-established risk factor for CNSTs, other studies found that viruses, infections, and harmful chemicals are related to CNSTs $[24,26]$. The period effect in China and globally showed a downwards trend over time, and the decrease in China was significantly higher than that globally. This may be due to the implementation of the new rural cooperative medical system in China in 2000, which achieved full coverage by 2010 [27]. This measure greatly improves the accessibility of medical services by rural Chinese residents. The cohort effect showed an overall upwards trend before 1990, and the increase gradually slowed and then fluctuated after 1990. During this period, the effect of China's reform and opening up in the 1980s gradually appeared, and medical policies and medical conditions gradually increased [28]. The nutrition and health level of pregnant women gradually improved, the height of children and adolescents increased significantly, and the prevalence of malnutrition and anaemia was significantly decreased $[29,30]$. Health status during early life has a strong impact on the future development of CNSTs. With the continuous progress of health in China, the health literacy of residents has also been steadily improved. In 2016, the health literacy level of Chinese residents was $11.58 \%$, higher than $6.48 \%$ in 2008 [31]. However, in recent years, environmental pollution and the increasing life pressure of young and middle-aged people may partially offset the protective effect against CNSTs.

Although CNST diagnosis and treatment had made some progress, there are still limitations. Although biopsies provide histological and genetic information, this method is invasive and risky and not conducive to longterm repeated sampling [32]. The most common noninvasive method is neurological imaging. MRI can show tumour progression slowly, but treatment-related MRI changes may be pseudo-progression. In addition, symptoms such as headache or epilepsy are often too common and nonspecific to indicate the need for further radiological examination, few headache patients suffer from CNSTs. Therefore, the feasible early diagnosis technology is very necessary to reduce the burden of CNSTs.

In our study, we analysed and compared the long-term epidemiological trend of CNSTs in China and globally and discussed the related factors. The incidence, mortality, and DALY rates showed that the implementation of health care policy, the improvement of health awareness, and the progression of medical technology have gradually reduced the average disease burden of CNSTs in the past 
30 years. However, due to China's huge population, the overall disease burden of CNSTs in China is still increasing. Therefore, in follow-up cancer treatment and control policies, we should continue to pay attention to key populations, early diagnosis technology, improvements in medical technology, and ways to reduce medical costs. Unfortunately, although GBD 2019 provides long-term CNSTs data, it does not collect CNSTs classification data, and we cannot perform further analysis, which requires other studies on tumour classification epidemiology.

\section{Acknowledgements}

We appreciate the works by all the investigators and collaborators from the GBD Network, without whom this study would not have been possible.

\section{Statement of Ethics}

Overall ethical approval was not required as this study did not require use of patient identifiers.

\section{Conflict of Interest Statement}

We declare no competing interests.

\section{Funding Sources}

No financial support was provided in the development of this manuscript. All our research data are extracted from GBD, which was partially funded by the Bill \& Melinda Gates Foundation, and the funders had no role in the study design, data analysis, data interpretation, or writing of the report in GBD.

\section{Author Contributions}

B.Z. and B.Y. designed the whole research; B.Z., X.W., and S.X. conducted the data collection; and B. Z. and X.W. analysed the data. B.Z., H.P., and B.Y. wrote the manuscript. B.Z., X.W., S.X., and B.Y. discussed the relevant results.

\section{Data Availability Statement}

All our research data are obtained from GBD 2019, and the website is http://ghdx.healthdata.org/gbd-results-tool.

\section{References}

1 Armstrong GT, Liu Q, Yasui Y, Huang S, Ness KK, Leisenring $\mathrm{W}$, et al. Longterm outcomes among adult survivors of childhood central nervous system malignancies in the Childhood Cancer Survivor Study. J Natl Cancer Inst. 2009;101(13): 946-58.

2 Gunn ME, Malila N, Lähdesmäki T, Arola M, Grönroos M, Matomäki J, et al. Late new morbidity in survivors of adolescent and youngadulthood brain tumors in Finland: a registry-based study. Neuro Oncol. 2015;17(10): 1412-8.

3 Yuan XG, Huang YR, Yu T, Xu Y, Liang Y, Zhang $\mathrm{XH}$, et al. Primary central nervous system lymphoma in China: a single-center retrospective analysis of 167 cases. Ann Hematol. 2020;99(1):93-104.

4 Giglio P, Gilbert MR. Neurologic complications of cancer and its treatment. Curr Oncol Rep. 2010;12(1):50-9.

5 Sung H, Ferlay J, Siegel RL, Laversanne M, Soerjomataram I, Jemal A, et al. Global cancer statistics 2020: GLOBOCAN estimates of incidence and mortality worldwide for $36 \mathrm{can}$ cers in 185 countries. CA Cancer J Clin. 2021; 71(3):209-49.

6 GBD 2016 Brain and Other CNS Cancer Collaborators. Global, regional, and national burden of brain and other CNS cancer, 19902016: a systematic analysis for the Global Burden of Disease Study 2016. Lancet Neurol. 2019;18(4):376-93.

The Epidemiological Trend of CNSTs in China and Globally
7 Ostrom QT, Patil N, Cioffi G, Waite K, Kruchko C, Barnholtz-Sloan JS. CBTRUS statistical report: primary brain and other central nervous system tumors diagnosed in the United States in 2013-2017. Neuro Oncol. 2020;22(12 Suppl 2):iv1-96.

8 Fuentes-Raspall R, Solans M, Roca-Barceló A, Vilardell L, Puigdemont M, Del Barco S, et al. Descriptive epidemiology of primary malignant and non-malignant central nervous tumors in Spain: results from the Girona cancer registry (1994-2013). Cancer Epidemiol. 2017;50(Pt A):1-8.

9 Stranjalis G, Kalamatianos T, Stavrinou LC, Mathios D, Koutsarnakis C, Tzavara C, et al. The Evangelismos hospital central nervous system tumor registry: analysis of 1,414 cases (1998-2009). Surg Neurol Int. 2013;4:23.

10 Lee $\mathrm{CH}$, Jung KW, Yoo H, Park S, Lee SH. Epidemiology of primary brain and central nervous system tumors in Korea. J Korean Neurosurg Soc. 2010;48(2):145-52.

11 Henderson R, Keeling P, French D, Smart D, Sullivan R, Lawler M. Cost-effectiveness of precision diagnostic testing for precision medicine approaches against non-small-cell lung cancer: a systematic review. Mol Oncol. 2021. Epub ahead of print.

12 Kayamba V, Heimburger DC, Morgan DR, Atadzhanov M, Kelly P. Exposure to biomass smoke as a risk factor for oesophageal and gastric cancer in low-income populations: a systematic review. Malawi Med J. 2017;29(2):212-7.
13 Lovrics O, Butt J, Lee Y, Lovrics P, Boudreau $\mathrm{V}$, Anvari $\mathrm{M}$, et al. The effect of bariatric surgery on breast cancer incidence and characteristics: a meta-analysis and systematic review. Am J Surg. 2021;222(4):715-22.

14 Zhang W, Zeng W, Jiang A, He Z, Shen X, Dong $X$, et al. Global, regional and national incidence, mortality and disability-adjusted life-years of skin cancers and trend analysis from 1990 to 2019: an analysis of the Global Burden of Disease Study 2019. Cancer Med. 2021;10(14):4905-22.

15 GBD 2019 Tobacco Collaborators. Spatial, temporal, and demographic patterns in prevalence of smoking tobacco use and attributable disease burden in 204 countries and territories, 1990-2019: a systematic analysis from the Global Burden of Disease Study 2019. Lancet. 2021;397(10292):2337-60.

16 Wu X, Zhu B, Xu S, Bi Y, Liu Y, Shi J. A cross country comparison for the burden of cardiovascular disease attributable to tobacco exposure in China, Japan, USA and world. BMC Public Health. 2020;20(1):888.

17 da Costa WL Jr, Oluyomi AO, Thrift AP. Trends in the Incidence of pancreatic adenocarcinoma in all 50 United States examined through an age-period-cohort analysis. JNCI Cancer Spectr. 2020;4(4):pkaa033.

18 Wu X, Zhu B, Zhou J, Bi Y, Xu S, Zhou B. The epidemiological trends in the burden of lung cancer attributable to PM2.5 exposure in China. BMC Public Health. 2021;21(1):737. 
19 Lai WW, Chung CH, Lin CN, Yang SC, Hwang JS, Wang JD. QALYs and medical costs saved from prevention of a cancer: analysis of nation-wide real-world data of Taiwan with lifetime horizon. J Formos Med Assoc. 2021. Epub ahead of print.

20 Wang Y, Zhao L, Gao L, Pan A, Xue H. Health policy and public health implications of obesity in China. Lancet Diabetes Endocrinol. 2021;9(7):446-61.

21 Eberle C, Kirchner MF, Herden R, Stichling S. Paternal metabolic and cardiovascular programming of their offspring: a systematic scoping review. PLoS One. 2020;15(12): e0244826.

22 Ramis R, Tamayo-Uria I, Gómez-Barroso D, López-Abente G, Morales-Piga A, Pardo Romaguera E, et al. Risk factors for central nervous system tumors in children: new findings from a case-control study. PLoS One. 2017; 12(2):e0171881.
23 Deng F, Lv JH, Wang HL, Gao JM, Zhou ZL. Expanding public health in China: an empirical analysis of healthcare inputs and outputs. Public health. 2017;142:73-84.

24 Michaud D, Batchelor T. Risk factors for brain tumors. Waltham: UpToDate Inc.; 2020 [cited $2020 \mathrm{Feb} 7]$. Available from: https:// www.uptodate.com/contents/risk-factorsfor-brain-tumors.

25 Yu MW, Quail DF. Immunotherapy for glioblastoma: current progress and challenge. Front Immunol. 2021;12:676301.

26 The American Cancer Society. Risk factors for brain and spinal cord tumors [cited 2020 May 5]. Available from: https://www.cancer. org/cancer/brain-spinal-cord-tumorsadults/causes-risks-prevention/risk-factors. html\#reference.

27 Li C, Hou Y, Sun M, Lu J, Wang Y, Li X, et al. An evaluation of China's new rural cooperative medical system: achievements and inadequacies from policy goals. BMC Public Health. 2015;15:1079.
28 Jiang J, Wang P. Health status in a transitional society: urban-rural disparities from a dynamic perspective in China. Popul Health Metr. 2018;16(1):22.

29 Dong CX, Yin SA. [The ten-year retrospect of nutrition and health status of pregnant women in China]. Zhonghua Yu Fang Yi Xue Za Zhi. 2018;52(1):94-100. Chinese.

30 Zhang X, Liu J, Jin Y, Yang S, Song Z, Jin L, et al. Folate of pregnant women after a nationwide folic acid supplementation in China. Matern Child Nutr. 2019;15(4):e12828.

31 Chen H, Lu M, Zhang Y, Lu B, You L, Dai M, et al. Trend analysis on morbidity and mortality of pancreatic cancer in China, 2005-2015. Chin J Epidemiol. 2021;42(5):794-800.

32 Cohen JV, Alomari AK, Vortmeyer AO, Jilaveanu LB, Goldberg SB, Mahajan A, et al. Melanoma brain metastasis pseudoprogression after pembrolizumab treatment. Cancer Immunol Res. 2016;4(3):179-82. 